Volume 3, Issue 1, 2016

\title{
Use of Medical Cannabis and its derivatives for the anticonvulsant property in Epileptic patients along with Ethical considerations: a Review
}

\author{
Article by Kavita Gupta \\ PhD Scholar at Texila American University, Guyana \\ E-mail:16kavitagupta@texilaconnect.com
}

\begin{abstract}
The present study focused on the use of medicinal cannabis and its derivatives for the treatment of epileptic seizures in children, as well as, adults. "Epilepsy" defined as a chronic non-communicable disorder of the brain had shown to affect people of all age groups. There were many conventional treatments discovered up till date to minimize and alleviate the symptoms produced as a result of epilepsy, yet none among them could provide the long term safety and efficacy of the interventional drug. On the other hand, increased resistance to conventional drug therapy is another issue which has made researchers to think for the alternative treatment for the long run. On an average, it had been estimated that approximately, 50 million people worldwide were suffering from epilepsy, which thus made it one of the most common neurological diseases globally. Epilepsy accounted for $0.75 \%$, of the global burden of disease due to premature mortality, lost work productivity, health care needs and unhealthy quality of life. In 2012, epilepsy was responsible for approximately 20. 6 million disability-adjusted life years (DALYs) lost. According to WHO survey, it was estimated that about $80 \%$ of the people suffering from epilepsy resided in low- and middleincome countries in which three fourths of people could not access the treatment due to costeffective or non-availability of the treatment in comparison to the people who availed the conventional treatment responded to only approximately $70 \%$ of the time. Besides, in many parts of the world, people with epilepsy and their families suffered a lot from stigma and discrimination therefore, making it a social economic burden for hampering the quality of life of the patient as well as, that of the patient caregivers. Several countries (including Canada, Netherlands, and Israel) and 23 of 50 states in the United States had permitted the use of cannabis for medicinal purposes, with or without undergoing a systematic medicines approval process. Epilepsy-related deaths are a significant public health problem, yet are poorly understood and often overlooked. Hence, people with epilepsy are two to three times more likely to die prematurely than people without the disease which is surely a matter of concern to both the health providers and the patient.
\end{abstract}

Keywords: 'cannabis', 'marijuana', 'cannabinoids', 'tetrahydrocannabinol', 'THC', 'Pediatric' 'cannabidiol', 'CBD', 'cannabidivarin', 'CBDV', 'seizures' and 'epilepsy'

\section{Abbreviations}

$D A L Y$ s $=$ disability-adjusted life years

$C B D=$ Cannabinoid

SUDEP = Sudden unexpected death in epilepsy

GNP $=$ Gross national Product

THC $=$ Tetrahydrocannabinol

EEG = Electroencephalogram

HIPAA = Health Insurance Portability and Accountability Act of 1996

WHO = World Health Organization

PAME = Partners against Mortality in Epilepsy 
South American Journal of Clinical Research

Volume 3, Issue 1, 2016

\section{Introduction}

It was observed that the month of November was declared as a "National Epilepsy Awareness Month"[1]. Epilepsy is defined as a brain (neurological) disorder that is accompanied by synchronized episodes of seizures of varied intensity and frequency with excessive electrical activity in the brain [1,2]. On persistent attack of seizures, itcaused disabling along with direct damage to the neural tissues [2]. Epilepsy was found to bemore prevalent amongst the children [1]. The greatest cause of epilepsy-related deaths, Sudden Unexpected Death in Epilepsy (SUDEP) was due to uncontrolled seizures [3]. Epilepsy was ascertained using diagnosis codes based on the International Classification of Diseases, Ninth Revision, Clinical Modification (ICD-9-CM) for epilepsy (345. 0, 345. 1, 345. 3-345. 9) and for seizures not otherwise specified (ICD- 9-CM 780.39) [1]. The positive predictive value of this group of diagnostic codes for an epilepsy diagnosis in children was $96.5 \%(95 \%$ confidence interval $[\mathrm{CI}]=88.1 \%-99.0 \%$ ) [1]. According to CDC study, it was observed that students aged 6-17 years with epilepsy were more likely to miss 11 or more days of school on an average as compared with the students who had health concerns other than epilepsy [4]. Apart from this, students with epilepsyfaced difficulties in school by none to limited participation in sports and other activities [4]. Epidemiologically, the disease had already affected about 2. 3 million adults and 450, 000 children in the United States in which only one third of the epileptic population had an access to the comprehensive care and approximately, more than 1 out of 1,000 people died of epilepsy each year $[1,3,5]$. About one third of the epileptic patients had anaccess to comprehensive care[1]. An Indian study conducted in 1998 calculated that the cost per patient of epilepsy treatment was as high as $88.2 \%$ of the country's per capita Gross National Product (GNP), and epilepsy-related costs, which included medical costs, travel, and lost work time, exceeded \$2. 6 billion/year [6]. It was estimated that approximately 6-10 million people in India lived with active Epilepsy, amongst which about only less than half were treated [7]. The high burden of Epilepsy in India affected patients financially along with disease-related burdens. [7]. The primary aim of the treatment is to potentially control seizures [2]. However, the best available treatments were often damaging while being partially effective [2]. Anti-epileptic drugs were found to be associated with adverse physical, behavioral and cognitive side-effects [2].

Cannabis sativais considered to be a medicinal plant that consisted of the dried flowers, leaves, and stems [8]. The hemp plant contained over 400 chemical compounds [8]. More than 80 cannabinoids (C21-containing compounds) unique to the cannabis plant had been identified [8]. Two major ingredients included:

- delta-9-Tetrahydrocannabinol, or THC, caused the psychoactive effects of "getting high. " [8].

- Cannabidiol, or CBD, did not cause psychoactive effects but had shown some positive effects on certain body systems and might potentially affected seizures. [9, $10]$.

Cannabidiol (CBD) is one of at least 60 active cannabinoids identified in cannabis. CBD was considered to had a wider scope of medical applications than tetrahydrocannabinol (THC), and appeared to have anti-convulsive action in animal models, as well as, clinical trials and surveys $[9,10]$. 


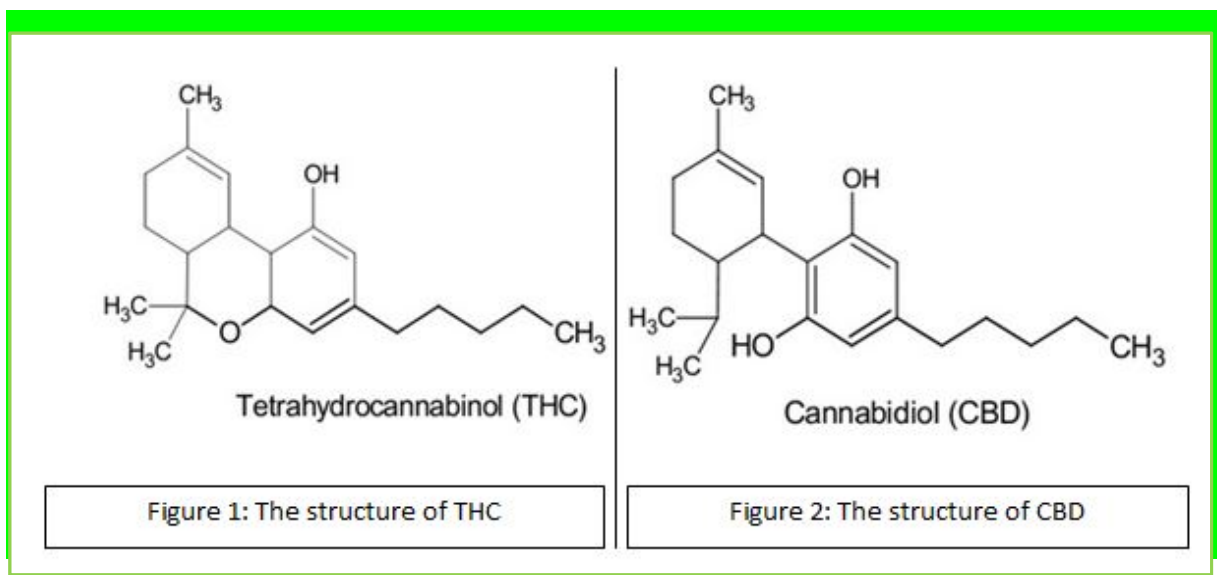

Fig 1. Molecular structure of Medicinal THC and CBD $[9,10]$

\section{Aim}

The main objective of this review was:

a. To understand the role of medicinal cannabis in the treatment of epilepsy,

b. To assess preclinical and clinical safety and efficacy of medicinal cannabis in reducing tonic seizures caused by epilepsy.

c. To understand the ethical issues and considerations behind the legal use of medicinal cannabis for the treatment purposes worldwide.

\section{Search methodology}

The core text that was focused throughout the article write up was 'Epilepsy and Cannabis'. All types of studies were included as there were very few clinical trials conducted in this regard. Moreover, children, Adolescents and Adults suffering from Epileptic seizures were included in this review. The methodology being adopted for the relevant literature review, searching the databases like, PUBMED, Cochrane Library, MEDLINE, EMBASE, Clinical trials. gov by using keywords such as, 'cannabis', 'marijuana', 'cannabinoids', 'tetrahydrocannabinol', 'THC', 'cannabidiol', 'CBD', 'cannabidivarin', 'CBDV', 'seizures' and 'epilepsy' was utilized. Moreover, a greater number of google searches were done to gather reliable and valid information from websites primarily focusing on safety and efficacy of medicinal cannabis use in epileptic patients. The search was confined to studies being published in English language. Moreover, PRISMA methodology was adopted for inclusion of relevant studies.

\section{Prisma flowchart methodology}


South American Journal of Clinical Research

Volume 3, Issue 1, 2016

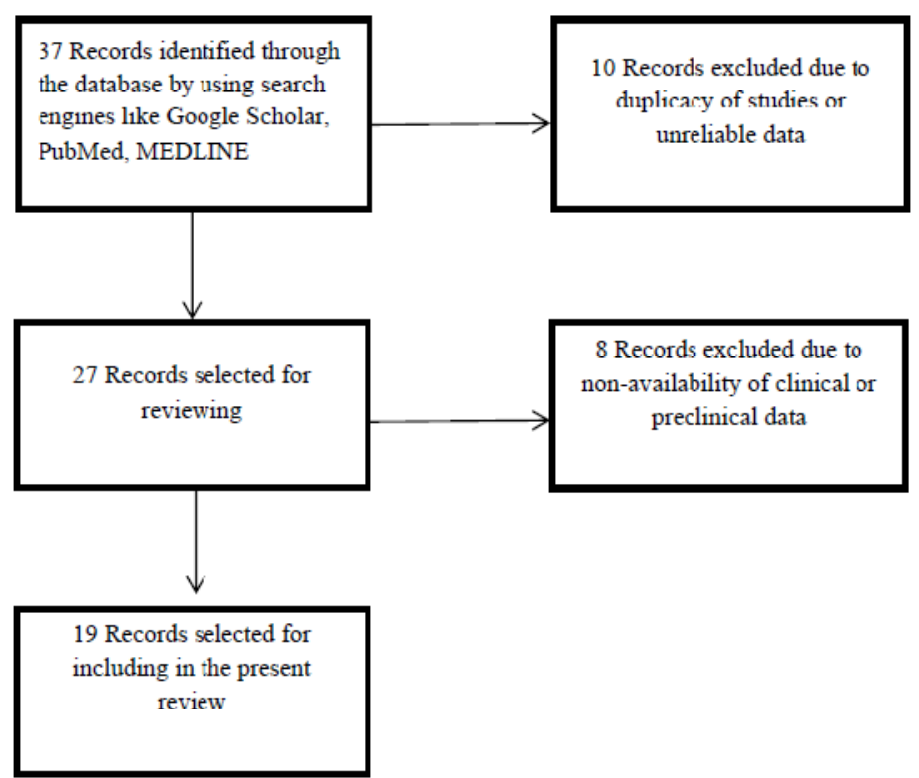

Fig 2. PRISMA FLOWCHART For Selection of Preclinical and Clinical Studies. 


\section{Description of studies (Clinical setting in humans)}

\begin{tabular}{|c|c|c|c|c|c|c|c|c|}
\hline $\begin{array}{l}\text { S. } \\
\text { No }\end{array}$ & Study & $\begin{array}{l}\text { Indicatio } \\
\text { n }\end{array}$ & Population & $\begin{array}{l}\text { Study } \\
\text { Design }\end{array}$ & $\begin{array}{l}\text { Intervention } \\
+ \\
\text { Route of } \\
\text { Administrati } \\
\text { on }\end{array}$ & \begin{tabular}{|l|} 
Results \\
\end{tabular} & Adverse Events & Significance \\
\hline 1. & \begin{tabular}{|l} 
Press et al. \\
2015 \\
{$[11]$}
\end{tabular} & Epilepsy & $\begin{array}{l}75 \text { children } \\
\text { with Epilepsy }\end{array}$ & $\begin{array}{l}\text { Open Label } \\
\text { Study }\end{array}$ & $\begin{array}{l}\text { Cannabis } \\
\text { orally }\end{array}$ & $\begin{array}{l}\text { Reduction and } \\
\text { improvement in } \\
\text { seizure control along } \\
\text { with improved motor } \\
\text { skills. }\end{array}$ & $\begin{array}{l}\text { somnolence/fatigue } \\
\text { developmental } \\
\text { regression, } \\
\text { abnormal } \\
\text { movements, }\end{array}$ & $\begin{array}{l}\text { Well tolerated } \\
\text { among children }\end{array}$ \\
\hline 2. & $\begin{array}{l}\text { Hamerle, M. } \\
\text { et al. } \\
2014 \\
{[12]}\end{array}$ & Epilepsy & $\begin{array}{l}310 \text { Epilepsy } \\
\text { patients }\end{array}$ & Survey & $\begin{array}{l}\text { Cannabis by } \\
\text { Inhalation }\end{array}$ & $\begin{array}{l}\text { Similar results as } \\
\text { produced by illicit } \\
\text { drug usage }\end{array}$ & $\begin{array}{l}\text { Mild to none side } \\
\text { effects }\end{array}$ & $\begin{array}{l}\text { Use of Cannabis did } \\
\text { not affect epilepsy }\end{array}$ \\
\hline 3. & \begin{tabular}{|l} 
Hegde, M. et \\
al. \\
2012 \\
{$[13]$}
\end{tabular} & Epilepsy & $\begin{array}{l}2 \text { patients with } \\
\text { epilepsy }\end{array}$ & $\begin{array}{l}\text { Uncontrolle } \\
\text { d case report }\end{array}$ & $\begin{array}{l}\text { Cannabis by } \\
\text { Inhalation }\end{array}$ & $\begin{array}{l}\text { Focal epilepsy } \\
\text { controlled }\end{array}$ & $\begin{array}{l}\text { On discontinuation } \\
\text { of Cannabis } \\
\text { medication, there } \\
\text { was a marked } \\
\text { increase in } \\
\text { frequency of } \\
\text { seizures. }\end{array}$ & Well tolerated \\
\hline 4. & \begin{tabular}{|l} 
Mortati, K. et \\
al. \\
2007 \\
{$[14]$}
\end{tabular} & Epilepsy & $\begin{array}{l}1 \text { patient with } \\
\text { cerebral palsy } \\
\text { and epilepsy }\end{array}$ & $\begin{array}{l}\text { Uncontrolle } \\
\text { d case report }\end{array}$ & $\begin{array}{l}\text { Cannabis by } \\
\text { Inhalation }\end{array}$ & \begin{tabular}{|l|} 
Reduction of \\
frequency of tonic \\
seizures
\end{tabular} & $\begin{array}{l}\text { No Serious adverse } \\
\text { events were } \\
\text { observed. }\end{array}$ & Well tolerated \\
\hline 5. & $\begin{array}{l}\text { Pelliccia, A. } \\
\text { et al. } \\
2005 \\
{[15]}\end{array}$ & Epilepsy & $\begin{array}{l}18 \text { children } \\
\text { with epilepsy }\end{array}$ & $\begin{array}{l}\text { Open Label } \\
\text { study }\end{array}$ & $\begin{array}{l}\text { Cannabidiol } \\
\text { Orally }\end{array}$ & $\begin{array}{l}\text { marked improvement } \\
\text { in both frequency and } \\
\text { intensity, awareness, } \\
\text { postural tone and }\end{array}$ & $\begin{array}{l}\text { no side effects of } \\
\text { such a severity }\end{array}$ & $\begin{array}{l}\text { Well tolerated } \\
\text { among children. }\end{array}$ \\
\hline
\end{tabular}


South American Journal of Clinical Research

Volume 3, Issue 1, 2016

\begin{tabular}{|c|c|c|c|c|c|c|c|c|}
\hline & & & & & & $\begin{array}{l}\text { speaking ability, and } \\
\text { attention-behavioural } \\
\text { improvement thereby } \\
\text { reduced } \\
\text { hospitalization. . }\end{array}$ & & \\
\hline 6. & $\begin{array}{l}\text { Cunha, J. M. } \\
\text { et al. } \\
1980 \\
{[16]}\end{array}$ & Epilepsy & $\begin{array}{l}15 \text { patients } \\
\text { with epilepsy }\end{array}$ & $\begin{array}{l}\text { Controlled } \\
\text { study }\end{array}$ & $\begin{array}{l}\text { Cannabidiol } \\
\text { Orally 200- } \\
300 \text { mg per } \\
\text { day + standard } \\
\text { medication } \\
\end{array}$ & $\begin{array}{l}\text { Partial reduction in } \\
\text { seizures. }\end{array}$ & no signs of toxicity & Well tolerated. \\
\hline 7. & $\begin{array}{l}\text { Consroe, } \mathrm{P} . \\
\text { F. et al. } \\
1975 \\
{[17]}\end{array}$ & Epilepsy & $\begin{array}{l}1 \text { patient with } \\
\text { epilepsy }\end{array}$ & $\begin{array}{l}\text { Uncontrolle } \\
\text { d case report }\end{array}$ & $\begin{array}{l}\text { Cannabis by } \\
\text { Inhalation ( } 2-5 \\
\text { cannabis } \\
\text { cigarettes per } \\
\text { day + standard } \\
\text { medication) }\end{array}$ & $\begin{array}{l}\text { Controlled seizures } \\
\text { for a short duration of } \\
\text { period. }\end{array}$ & no signs of toxicity & $\begin{array}{l}\text { Well tolerated when } \\
\text { used in conjunction } \\
\text { with standard } \\
\text { treatment. }\end{array}$ \\
\hline 8. & $\begin{array}{l}\text { Devinsky } \\
\text { Orrin, et al. } \\
2015 \\
{[18]}\end{array}$ & Epilepsy & $\begin{array}{l}214 \text { patients } \\
\text { (Chidren }+ \\
\text { Adults aged } \\
\text { from 1-30 } \\
\text { years) with } \\
\text { Epilepsy }\end{array}$ & $\begin{array}{l}\text { Open Label } \\
\text { Study }\end{array}$ & $\begin{array}{l}\text { Cannabidiol } \\
\text { Orally at } 2-5 \\
\mathrm{mg} / \mathrm{kg} \text { per } \\
\text { day, }\end{array}$ & $\begin{array}{l}\text { reduced seizure } \\
\text { frequency }\end{array}$ & $\begin{array}{l}\text { Reduction in } \\
\text { appetite, diarrhoea, }\end{array}$ & $\begin{array}{l}\text { Safe and well } \\
\text { tolerated }\end{array}$ \\
\hline 9. & $\begin{array}{l}\text { Gedde, M. \& } \\
\text { Maa, E. } \\
2013 \\
{[19]}\end{array}$ & Epilepsy & $\begin{array}{l}13 \text { children } \\
\text { with Epilepsy }\end{array}$ & $\begin{array}{l}\text { Observation } \\
\text { al study } \\
\text { (Survey) }\end{array}$ & $\begin{array}{l}\text { Realm } \\
\text { Oil treatment } \\
\text { at therate of } 4 \\
\text { to } 12 \\
\mathrm{mg} / \mathrm{kg} / \text { day, in } \\
2 \text { or } 3 \text { divided } \\
\text { doses }\end{array}$ & Reduction in seizures & $\begin{array}{l}\text { sedation and } \\
\text { unsteadiness, }\end{array}$ & Well tolerated \\
\hline 10. & $\begin{array}{l}\text { Lorenz, R. } \\
2003\end{array}$ & Epilepsy & $\begin{array}{l}8 \text { children } \\
\text { with epilepsy }\end{array}$ & $\begin{array}{l}\text { Uncontrolle } \\
\text { d case report }\end{array}$ & $\begin{array}{l}\text { Delta-9-THC } \\
\text { (Tetrahydroca }\end{array}$ & $\begin{array}{l}\text { Positive psychotropic } \\
\text { effects. }\end{array}$ & $\begin{array}{l}\text { No serious side- } \\
\text { effects }\end{array}$ & Well tolerated \\
\hline
\end{tabular}




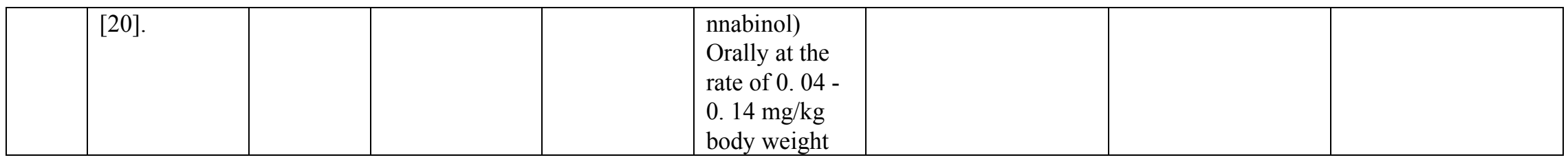

\section{Description of preclinical settings}

\begin{tabular}{|c|c|c|c|c|c|c|c|c|}
\hline $\begin{array}{l}\text { S. } \\
\text { No. }\end{array}$ & Study & $\begin{array}{l}\text { Indicatio } \\
\mathbf{n}\end{array}$ & Population & $\begin{array}{l}\text { Study } \\
\text { Design }\end{array}$ & $\begin{array}{l}\text { Intervention } \\
+ \\
\text { Route of Administration }\end{array}$ & Results & Adverse Events & Significance \\
\hline 1. & $\begin{array}{l}\text { Johnson, D. D. } \\
\text { et al. } \\
1975 \\
{[21] \text {. }}\end{array}$ & Epilepsy & $\begin{array}{l}\text { Epileptic } \\
\text { chickens }\end{array}$ & $\begin{array}{l}\text { Preclini } \\
\text { cal } \\
\text { study }\end{array}$ & $\begin{array}{l}\text { Intravenous administration } \\
\text { of delta9- } \\
\text { tetrahydrocannabinol } \\
\text { (delta9-THC) }\end{array}$ & $\begin{array}{l}\text { reduction in } \\
\text { frequency of } \\
\text { tonic seizures }\end{array}$ & $\begin{array}{l}\text { No serious side } \\
\text { effects }\end{array}$ & $\begin{array}{l}\text { Safe and well } \\
\text { tolerated }\end{array}$ \\
\hline 2. & $\begin{array}{l}\text { Florek- } \\
\text { Luszczki, M. } \\
\text { et al. } \\
2015 \\
{[22]}\end{array}$ & Epilepsy & $\begin{array}{l}\text { albino } \\
\text { Swiss mice }\end{array}$ & $\begin{array}{l}\text { Preclini } \\
\text { cal } \\
\text { study }\end{array}$ & $\begin{array}{l}\text { Intraperitoneal Injection of } \\
\text { WIN } 55,212-2 \text { mesylate } \\
\text { by a current ( } 32 \mathrm{~mA}, 6 \mathrm{~Hz} \text {, } \\
\text { 3s stimulus duration) }\end{array}$ & $\begin{array}{l}\text { Reduction in } \\
\text { tonic seizures. }\end{array}$ & $\begin{array}{l}\text { No serious side } \\
\text { effects }\end{array}$ & $\begin{array}{l}\text { Better } \\
\text { tolerated }\end{array}$ \\
\hline 3. & $\begin{array}{l}\text { Liu, H. et al. } \\
2015 \\
{[23]}\end{array}$ & Epilepsy & Mice & $\begin{array}{l}\text { Preclini } \\
\text { cal } \\
\text { study }\end{array}$ & $\begin{array}{l}\text { Administration of essential } \\
\text { oil in kainic acid-induced } \\
\text { seizure mice model. }\end{array}$ & $\begin{array}{l}\text { decreased seizure } \\
\text { activity score }\end{array}$ & $\begin{array}{l}\text { No serious side } \\
\text { effects }\end{array}$ & $\begin{array}{l}\text { Safe to use as } \\
\text { a protector }\end{array}$ \\
\hline 4. & $\begin{array}{l}\text { Izquierdo, I. et } \\
\text { al. }\end{array}$ & Epilepsy & $\begin{array}{l}\text { Convulsant } \\
\text { hippocamp }\end{array}$ & $\begin{array}{l}\text { Preclini } \\
\text { cal }\end{array}$ & $\begin{array}{l}5 \mathrm{mg} / \mathrm{kg} \text { of cannabidiol } \\
\text { administered by }\end{array}$ & $\begin{array}{l}\text { Reduced } \\
\text { susceptibility }\end{array}$ & None & $\begin{array}{l}\text { effectively } \\
\text { blocked the }\end{array}$ \\
\hline
\end{tabular}


South American Journal of Clinical Research

Volume 3, Issue 1, 2016

\begin{tabular}{|c|c|c|c|c|c|c|c|c|}
\hline & $\begin{array}{l}1973 \\
{[24] .}\end{array}$ & & $\begin{array}{l}\text { al } \\
\text { discharges } \\
\text { model of } \\
\text { Rat }\end{array}$ & study & $\begin{array}{l}\text { intraperitoneal injection } \\
\text { and topical application }\end{array}$ & & & $\begin{array}{l}\text { release of } \mathrm{K}+ \\
\text { from the } \\
\text { hippocampus }\end{array}$ \\
\hline 5. & $\begin{array}{l}\text { Consroe, P. , } \\
\text { \& Wolkin, A. } \\
1977 \\
{[25] .}\end{array}$ & Epilepsy & $\begin{array}{l}\text { Electroshoc } \\
\text { k-induced } \\
\text { seizure } \\
\text { model of } \\
\text { Rats }\end{array}$ & $\begin{array}{l}\text { Preclini } \\
\text { cal } \\
\text { study }\end{array}$ & Cannabidiol given orally & $\begin{array}{l}\text { Reduction of } \\
\text { tonic seizures }\end{array}$ & None & $\begin{array}{l}\text { Well } \\
\text { tolerated }\end{array}$ \\
\hline 6. & $\begin{array}{l}\text { Consroe, P. et } \\
\text { al. } \\
1982 \\
{[26] \text {. }}\end{array}$ & Epilepsy & $\begin{array}{l}\text { Electroshoc } \\
\text { k-induced } \\
\text { seizure } \\
\text { transcornea } \\
1 \text { model of } \\
\text { Mice }\end{array}$ & $\begin{array}{l}\text { Preclini } \\
\text { cal } \\
\text { study }\end{array}$ & $\begin{array}{l}\text { cannabidiol drug } \\
\text { administered by } \\
\text { intraperitoneal injection } \\
\text { and topical application }\end{array}$ & $\begin{array}{l}\text { Reduction of } \\
\text { tonic seizures }\end{array}$ & None & $\begin{array}{l}\text { Inhibited } \\
\text { seizure }\end{array}$ \\
\hline 7. & $\begin{array}{l}\text { Consroe, P. et } \\
\text { al. } \\
1982 \\
{[26] .}\end{array}$ & Epilepsy & $\begin{array}{l}\text { Seizures } \\
\text { induced by } \\
\text { strychnine } \\
\text { sulphate } \\
\text { model of } \\
\text { Mice }\end{array}$ & $\begin{array}{l}\text { Preclini } \\
\text { cal } \\
\text { study }\end{array}$ & $\begin{array}{l}\text { cannabidiol drug } \\
\text { administered by } \\
\text { intraperitoneal injection } \\
\text { and topical application }\end{array}$ & $\begin{array}{l}\text { No protection } \\
\text { against seizures } \\
\text { or death }\end{array}$ & None & $\begin{array}{l}\text { Inhibited } \\
\text { seizure }\end{array}$ \\
\hline 8. & $\begin{array}{l}\text { Consroe, P. et } \\
\text { al. } \\
1982 \\
{[26] .}\end{array}$ & Epilepsy & $\begin{array}{l}\text { Picrotoxin- } \\
\text { induced } \\
\text { seizures } \\
\text { model of } \\
\text { mice }\end{array}$ & $\begin{array}{l}\text { Preclini } \\
\text { cal } \\
\text { study }\end{array}$ & $\begin{array}{l}\text { cannabidiol drug } \\
\text { administered by } \\
\text { intraperitoneal injection } \\
\text { and topical application }\end{array}$ & $\begin{array}{l}\text { Reduction of } \\
\text { tonic seizures }\end{array}$ & None & $\begin{array}{l}\text { Inhibited } \\
\text { seizure }\end{array}$ \\
\hline 9. & $\begin{array}{l}\text { Consroe, P. et } \\
\text { al. } \\
1982 \\
{[26] .}\end{array}$ & Epilepsy & $\begin{array}{l}\text { Seizures } \\
\text { induced by } \\
3- \\
\text { mercaptopr } \\
\text { opionic } \\
\end{array}$ & $\begin{array}{l}\text { Preclini } \\
\text { cal } \\
\text { study }\end{array}$ & $\begin{array}{l}\text { cannabidiol drug } \\
\text { administered by } \\
\text { intraperitoneal injection } \\
\text { and topical application }\end{array}$ & $\begin{array}{l}\text { Reduction of } \\
\text { tonic seizures }\end{array}$ & None & $\begin{array}{l}\text { Inhibited } \\
\text { seizure }\end{array}$ \\
\hline
\end{tabular}


South American Journal of Clinical Research Volume 3, Issue 1, 2016

\begin{tabular}{|c|c|c|c|c|c|c|c|c|}
\hline & & & $\begin{array}{l}\text { acid model } \\
\text { of mice }\end{array}$ & & & & & \\
\hline 10. & $\begin{array}{l}\text { Consroe, P. et } \\
\text { al. } \\
1982 \\
{[26] .}\end{array}$ & Epilepsy & $\begin{array}{l}\text { Pentylenete } \\
\text { trazol- } \\
\text { induced } \\
\text { seizures } \\
\text { model of } \\
\text { mice }\end{array}$ & $\begin{array}{l}\text { Preclini } \\
\text { cal } \\
\text { study }\end{array}$ & $\begin{array}{l}\text { cannabidiol drug } \\
\text { administered by } \\
\text { intraperitoneal injection } \\
\text { and topical application }\end{array}$ & $\begin{array}{l}\text { Reduction of } \\
\text { tonic seizures }\end{array}$ & None & $\begin{array}{l}\text { Inhibited } \\
\text { seizure }\end{array}$ \\
\hline 11. & $\begin{array}{l}\text { Consroe, P. et } \\
\text { al. } \\
1982 \\
{[26] .}\end{array}$ & Epilepsy & $\begin{array}{l}\text { Seizures } \\
\text { induced by } \\
\text { isonicotinic } \\
\text { acid } \\
\text { hydrazide } \\
\text { model of } \\
\text { mice }\end{array}$ & $\begin{array}{l}\text { Preclini } \\
\text { cal } \\
\text { study }\end{array}$ & $\begin{array}{l}\text { cannabidiol drug } \\
\text { administered by } \\
\text { intraperitoneal injection } \\
\text { and topical application }\end{array}$ & $\begin{array}{l}\text { Reduction of } \\
\text { tonic seizures }\end{array}$ & None & $\begin{array}{l}\text { Inhibited } \\
\text { seizure }\end{array}$ \\
\hline 12. & $\begin{array}{l}\text { Consroe, P. et } \\
\text { al. } \\
1982 \\
{[26] .}\end{array}$ & Epilepsy & $\begin{array}{l}\text { Bicuculline } \\
\text {-induced } \\
\text { seizures } \\
\text { model of } \\
\text { mice }\end{array}$ & $\begin{array}{l}\text { Preclini } \\
\text { cal } \\
\text { study }\end{array}$ & $\begin{array}{l}\text { cannabidiol drug } \\
\text { administered by } \\
\text { intraperitoneal injection } \\
\text { and topical application }\end{array}$ & $\begin{array}{l}\text { Reduction of } \\
\text { tonic seizures }\end{array}$ & None & $\begin{array}{l}\text { Inhibited } \\
\text { seizure }\end{array}$ \\
\hline 13. & $\begin{array}{l}\text { Jones, N. A. et } \\
\text { al. } \\
2010 \\
{[27] .}\end{array}$ & Epilepsy & $\begin{array}{l}\text { Pentylenete } \\
\text { trazol- } \\
\text { induced } \\
\text { seizures } \\
\text { model of } \\
\text { rats }\end{array}$ & $\begin{array}{l}\text { Preclini } \\
\text { cal } \\
\text { study }\end{array}$ & $\begin{array}{l}\text { cannabidiol drug }(1,10, \\
\text { and } 100 \mathrm{mg} / \mathrm{kg}) \text { by } \\
\text { multielectrode array }\end{array}$ & $\begin{array}{l}\text { Seizure and } \\
\text { mortality } \\
\text { reduction }\end{array}$ & None & $\begin{array}{l}\text { CBD } \\
\text { inhibited } \\
\text { epileptiform } \\
\text { activity in } \\
\text { vitro and } \\
\text { seizure } \\
\text { severity in } \\
\text { vivo }\end{array}$ \\
\hline 14. & $\begin{array}{l}\text { Jones, N. A. et } \\
\text { al. }\end{array}$ & Epilepsy & $\begin{array}{l}\text { Pilocarpine } \\
\text {-induced }\end{array}$ & $\begin{array}{l}\text { Preclini } \\
\text { cal }\end{array}$ & $\begin{array}{l}\text { pure CBD }(1,10 \text { and } 100 \\
\mathrm{mg} / \mathrm{kg}) \text { administered by }\end{array}$ & Seizure reduction & None & $\begin{array}{l}\text { CBD as a } \\
\text { therapeutic }\end{array}$ \\
\hline
\end{tabular}


South American Journal of Clinical Research

Volume 3, Issue 1, 2016

\begin{tabular}{|c|c|c|c|c|c|c|c|c|}
\hline & $\begin{array}{l}2012 \\
{[28] .}\end{array}$ & & $\begin{array}{l}\text { seizures } \\
\text { model of } \\
\text { rats }\end{array}$ & study & $\begin{array}{l}\text { intraperitoneal injection } \\
\text { and topical application }\end{array}$ & & & $\begin{array}{l}\text { candidate for } \\
\text { a diverse } \\
\text { range of } \\
\text { human } \\
\text { epilepsies. }\end{array}$ \\
\hline 15 . & $\begin{array}{l}\text { Jones, N. A. et } \\
\text { al. } \\
2012 \\
{[28] .}\end{array}$ & Epilepsy & $\begin{array}{l}\text { Partial } \\
\text { seizures } \\
\text { induced by } \\
\text { intraventric } \\
\text { ular } \\
\text { penicillin } \\
\text { model of } \\
\text { rats }\end{array}$ & $\begin{array}{l}\text { Preclini } \\
\text { cal } \\
\text { study }\end{array}$ & $\begin{array}{l}\text { pure CBD }(1,10 \text { and } 100 \\
\mathrm{mg} / \mathrm{kg}) \text { administered by } \\
\text { intraperitoneal injection } \\
\text { and topical application }\end{array}$ & Seizure reduction & None & $\begin{array}{l}\text { CBD as a } \\
\text { therapeutic } \\
\text { candidate for } \\
\text { a diverse } \\
\text { range of } \\
\text { human } \\
\text { epilepsies. }\end{array}$ \\
\hline 16. & $\begin{array}{l}\text { Shirazi-zand, } \\
\text { Z. et al. } \\
2013 \\
{[29] .}\end{array}$ & Epilepsy & $\begin{array}{l}\text { Electroshoc } \\
\mathrm{k} \text {-induced } \\
\text { seizure } \\
\text { model of } \\
\text { mice }\end{array}$ & $\begin{array}{l}\text { Preclini } \\
\text { cal } \\
\text { study }\end{array}$ & $\begin{array}{l}\text { Intracerebroventricular } \\
\text { administration of } \\
\text { cannabidiol }\end{array}$ & $\begin{array}{l}\text { Increased } \\
\text { threshold }\end{array}$ & None & $\begin{array}{l}\text { Well } \\
\text { tolerated }\end{array}$ \\
\hline 17. & $\begin{array}{l}\text { Shirazi-zand, } \\
\text { Z. et al. } \\
2013 \\
{[29] .}\end{array}$ & Epilepsy & $\begin{array}{l}\text { Pentylenete } \\
\text { trazol- } \\
\text { induced } \\
\text { seizures } \\
\text { model of } \\
\text { mice }\end{array}$ & $\begin{array}{l}\text { Preclini } \\
\text { cal } \\
\text { study }\end{array}$ & $\begin{array}{l}\text { Intracerebroventricular } \\
\text { administration of } \\
\text { cannabidiol }\end{array}$ & $\begin{array}{l}\text { Increased } \\
\text { threshold }\end{array}$ & None & $\begin{array}{l}\text { Well } \\
\text { tolerated }\end{array}$ \\
\hline
\end{tabular}




\section{Data interpretation}

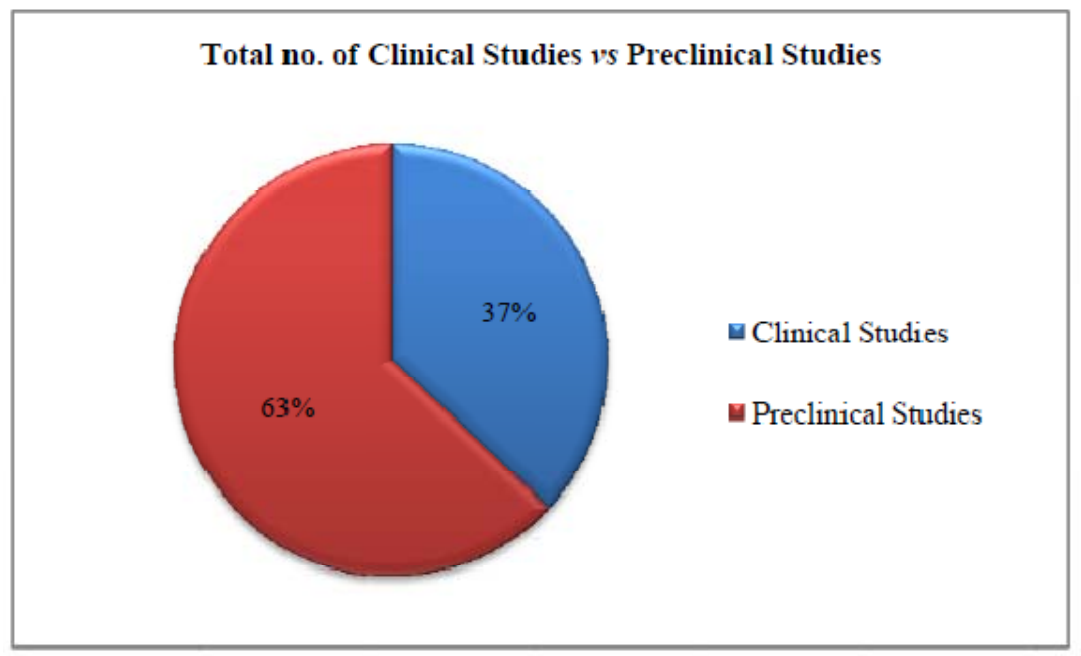

Fig 3. Clinical Studies vs. Preclinical Studies [11-29].

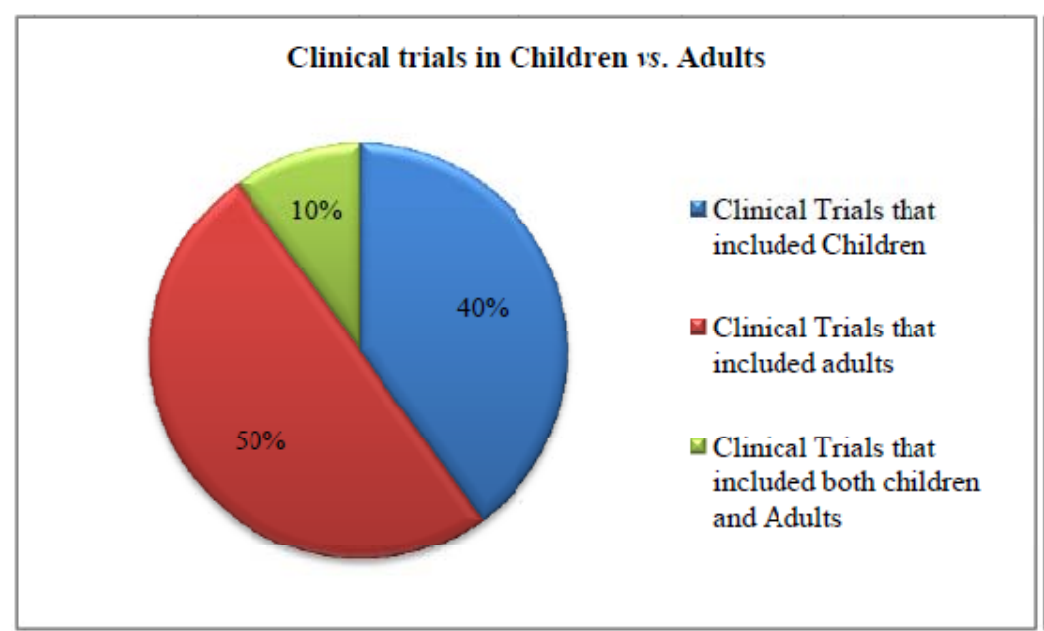

Fig. 4. Clinical Trials in Children vs. Clinical Trials in Adults [11-20].

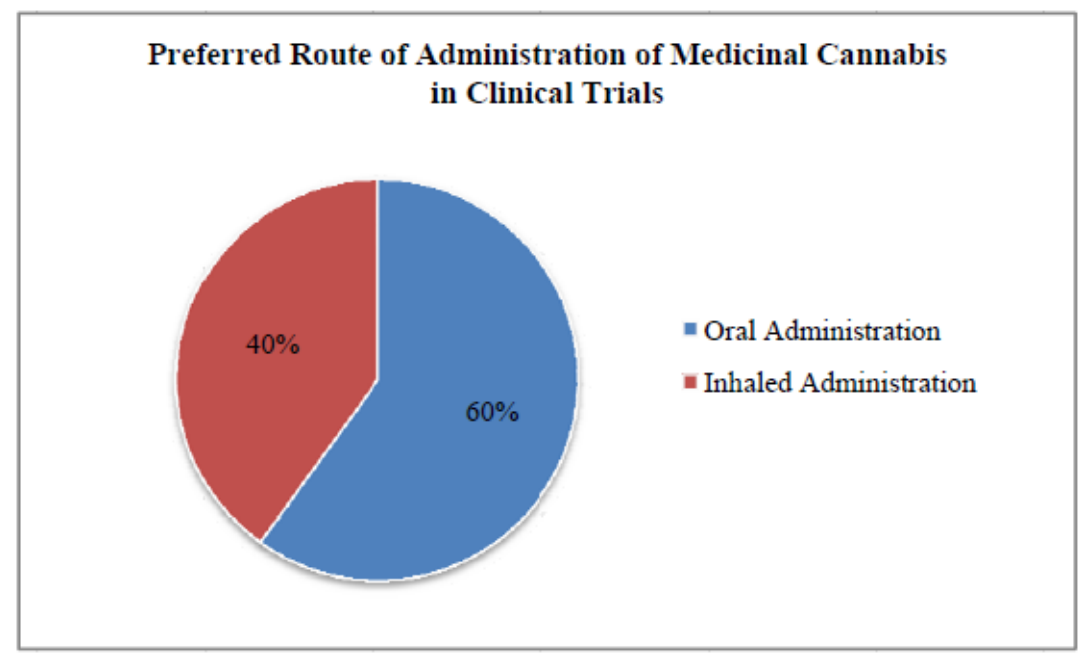

Fig 5. Preferred Route of Administration of Medicinal Cannabis in Clinical Trials [11-20]. 
South American Journal of Clinical Research

Volume 3, Issue 1, 2016

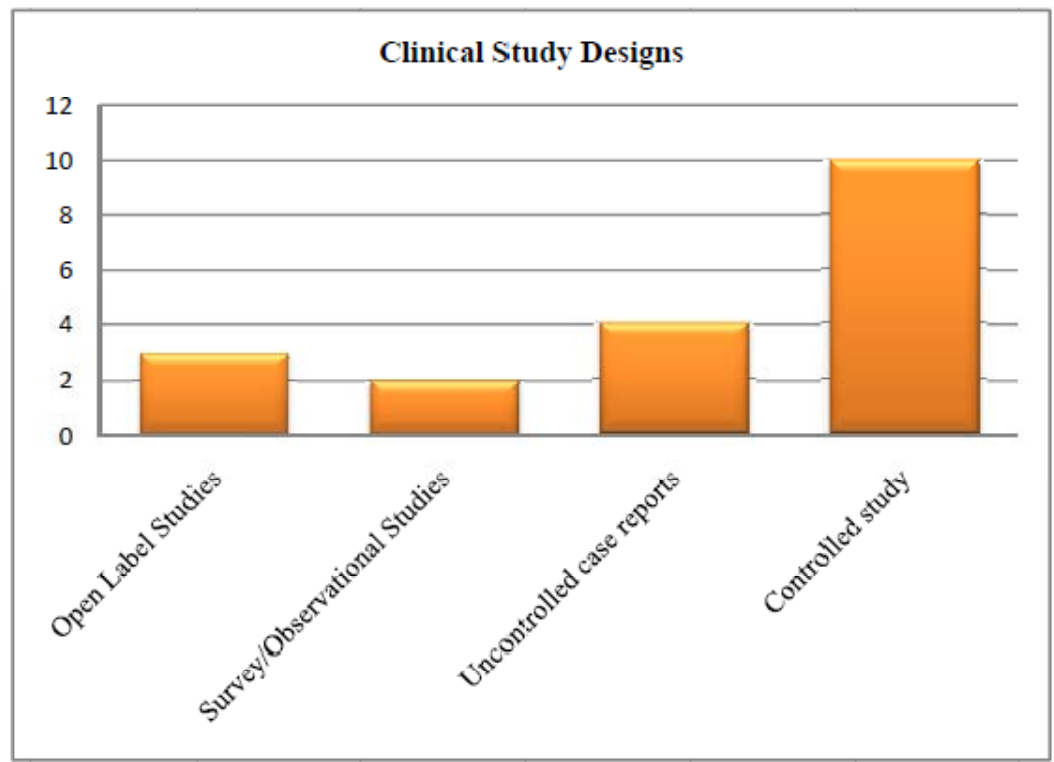

Fig 6. Clinical Study Designs [11-20]

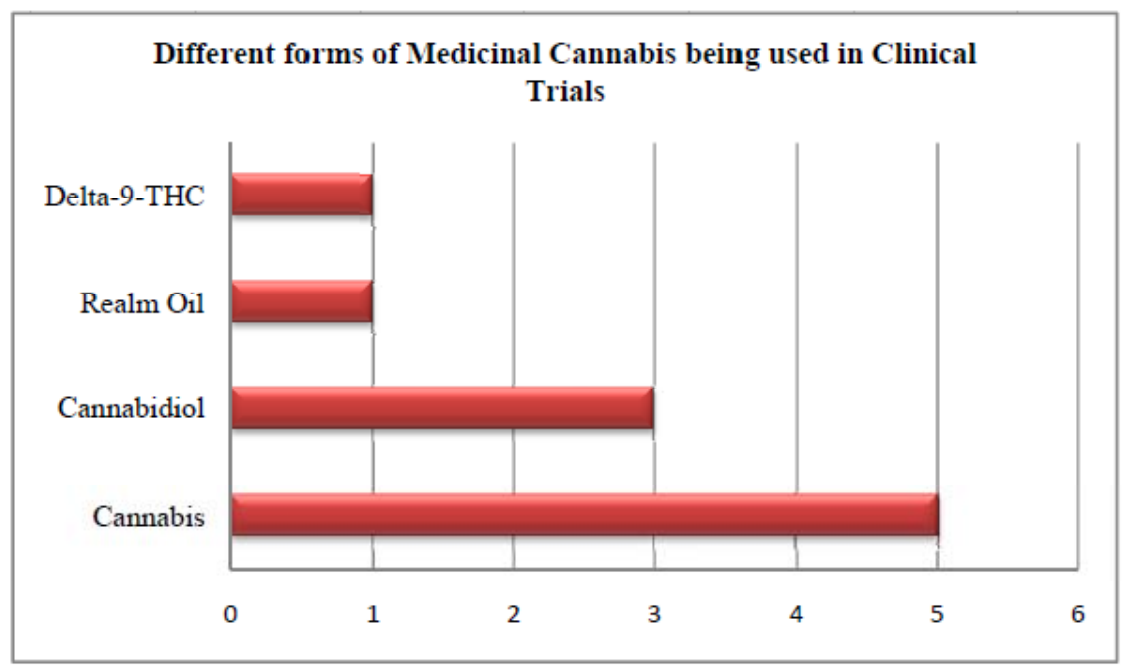

Fig 7. Different forms of Medicinal Cannabis being used in Clinical Trials [11-20].

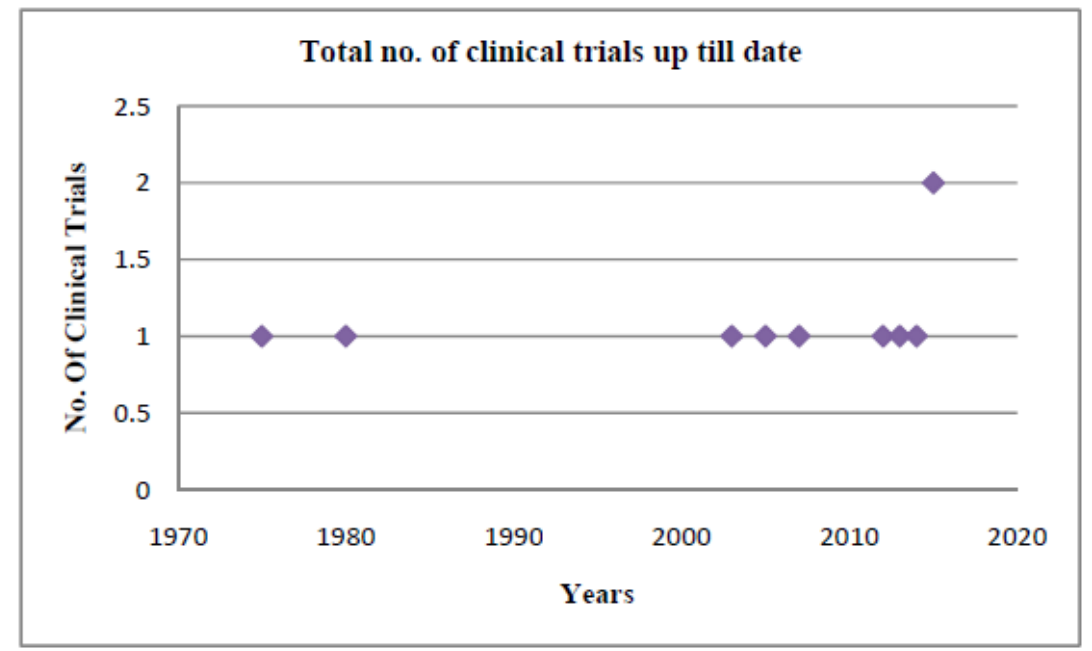


Fig 8. Total no. of clinical trials up till date [11-20].

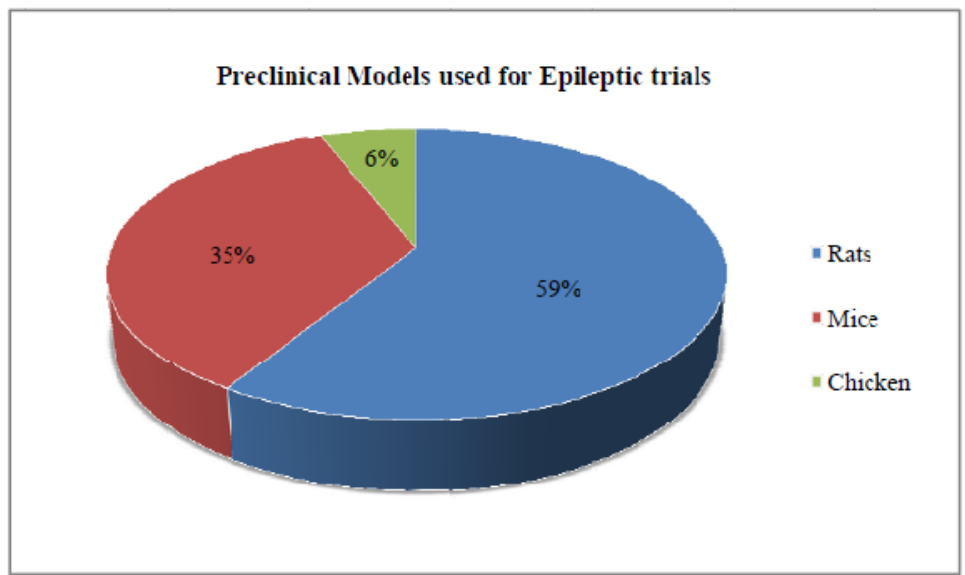

Fig 9. Preclinical Models used for Epileptic trials [21-29].

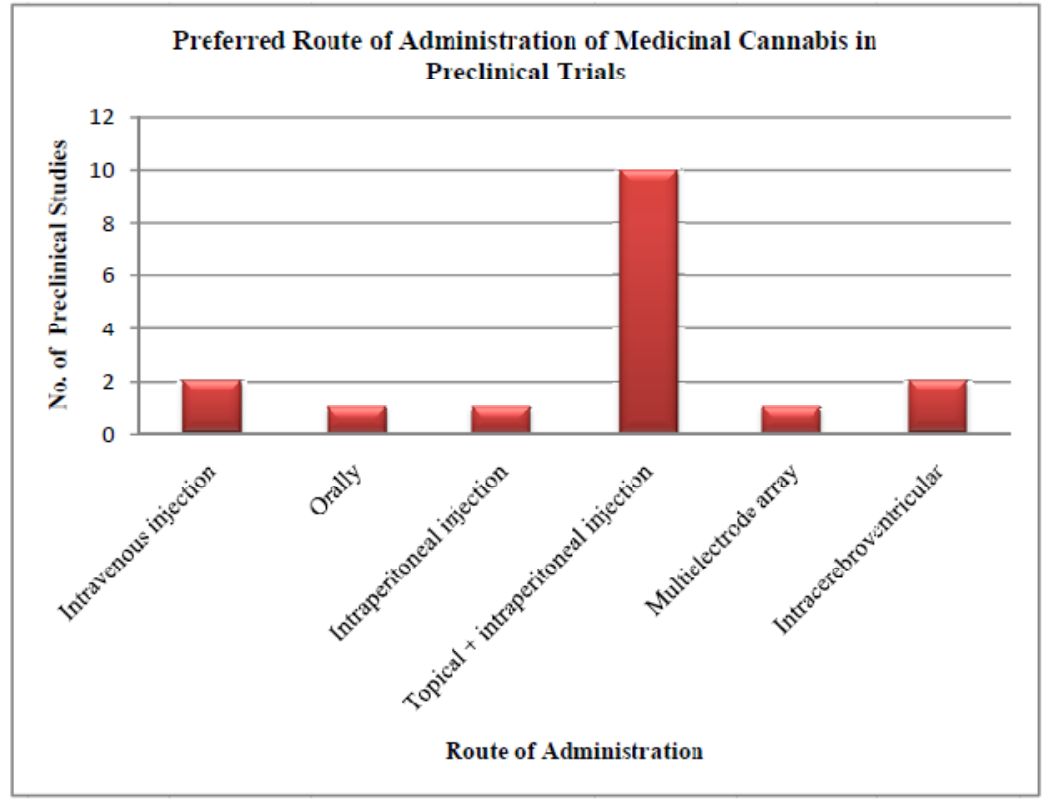

Fig 10. Preferred Route of Administration of Medicinal Cannabis in Preclinical Trials [21-29]

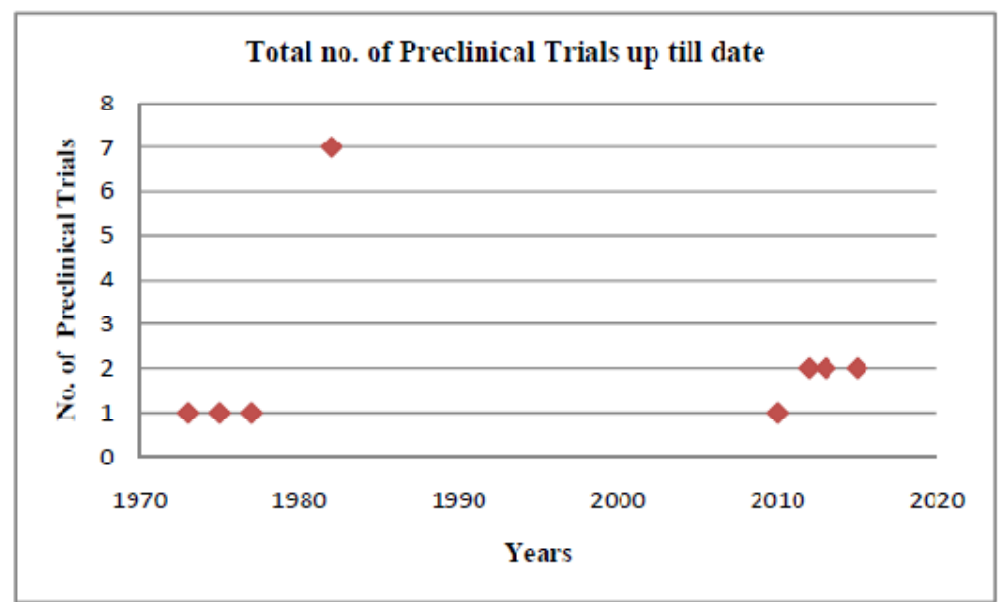

Fig 11. Total no. of Preclinical Trials up till date [21-29]. 
South American Journal of Clinical Research

Volume 3, Issue 1, 2016

\section{Ethical considerations}

Medicinal Cannabis had remained lodged in Schedule I under the 1970 Controlled Substances Act of the U. S. federal Controlled Substances Act, meaning it has "no currently accepted medical use and a high potential for abuse, due to which cannabis was highly restricted and prohibited to be used for clinical research [30]. However, the extensive literature research on therapeutic cannabis had been reviewed over the past several years by various governmental and academic bodies, including the U. S. National Academy of Sciences-Institute of Medicine, UK House of Lords, and Canadian Senate Committee on Illegal Drugs which reported in the confirmation of the therapeutic value of medicinal cannabis [31]. From the above review, it could be concluded that there were some ethical issues in Clinical Practice of Medicinal Cannabis. The doctors cannot dispense the drug in any form. The doctors could recommend, however, are not allowed to prescribe medicinal cannabis [8]. The goal of palliative care, is to improve the quality of life for a patient by preventing or relieving symptoms of disease and side effects of treatments [8]. On one side, the use of medicinal cannabis contributed to the good effects it could have produced by supporting the factor of 'Beneficience' in accord of ethical conduct to practice medicine. But, on the other hand, it violated the 'Non-malfeasance'factor which was meant for doing no harm to the patient while conducting clinical trial ethically[8]. On the contrary, both the factors were found to be very contradictory and conflicting as well. The ethical question that arose was the existence of any practices that could control medicinal cannabis abuse, educational qualification for recommending medicinal therapeutic cannabis and integration of the treatment into the routine health assessment care [8].

\section{Discussion}

From the above literature reviewed, it could be suggested that anecdotal evidence was sufficient to support the fact that some children suffering from epilepsy and seizures got benefited up to some extent by the use of oral medicinal cannabis. This further provided an evidence-based approach for proposing and determining objective data and requirement of Randomized placebo-controlled trials. From ethical point of view, seriously ill patients (Children and adults) have the right to access effective therapies that improved their quality of life, however, if this right is denied then their dignity and respect might get harmed which ultimately might force them to go out of the way to access the medical care. On the other hand, the data collected from various sources pointed in a direction that none of the medicinal cannabinoid product was tested for its safety and efficacy for long term usage in children and adults suffering from epilepsy. Moreover, only a small number of case reports and surveys existed with conflicting results that included pediatric patients with epilepsy in greater number.

Review of clinical data interpretation: It was observed that there were comparatively less number of clinical trials as compared to preclinical trials conducted in the subsequent years from 1973 to 2015. During these span of years, it was observed that Medicinal Cannabis was used as an intervention in the age group of children suffering from epilepsy in comparison to the adults and adolescents suffering from epilepsy. Moreover, it was seen that oral Medicinal Cannabis and Cannibidiol were well tolerated as compared to inhaled Medicinal Cannabis amongst epileptic patients by reduction of tonic seizures and marked improvement in the frequency and intensity of seizure control, motor skills, awareness, postural tone, speaking ability with positive psychotropic effects on the epileptic patients suffering. However, the question of use of Medicinal Cannabis for its long-term use and effects to alleviate the symptoms of epilepsy in patients is still conflicting due to lack of evidence based trials. There was only one randomized controlled study being conducted which was considered to be a pilot study with small sample size, thus inhibiting safety and efficacy of Medicinal use of Cannabis in epilepsy. On the other hand, mild adverse effects were found to be associated with the intervention of medicinal Cannabis and its derivatives, 
that were, namely, fatigue, developmental regression, abnormal movements, appetite reduction, diarrhea, sedation and unsteadiness [11-20].

Review of preclinical data interpretation: During preclinical study review, it was observed that maximum number of preclinical trials was conducted on Rat models in comparison to few numbers of preclinical trials being conducted on Chicken model and Mice model. It was also predicted that the adverse effects associated with the intervention of medicinal Cannabis in animal models showed none to minimal side-effects which led to the evidence based fact that medicinal Cannabis and its derivatives could be used safely in humans in a clinical setting. The outcomes observed from the preclinical study of medicinal cannabis intervention were reduction in EEG activity, tonic seizures, and susceptibility with low mortality rate and increased threshold which further pointed towards the fact that the medicinal cannabis drug intervention was well-tolerated in animal models [21-29].

According to 'Partners Against Mortality in Epilepsy (PAME)', epilepsy affected 3 million Americans, creating a blind spot for caregivers that could prove devastating because epilepsy is often not listed as a cause of death on death certificates, making it difficult for public health officials to quantify and draw attention to the problem [32]. The lack of data also challenged to find a best alternative treatment to cure epilepsy both in children and adults. [32].

\section{Clinical trials pending}

Following are the awaited high methodology quality Prospective data for evaluating safety and efficacy of Medicinal Cannabis for the treatment of Epilepsy in children as well as, adult population. 
South American Journal of Clinical Research

Volume 3, Issue 1, 2016

Description of pending clinical trials

\begin{tabular}{|c|c|c|c|c|c|c|}
\hline $\begin{array}{l}\text { S. } \\
\text { No. }\end{array}$ & $\begin{array}{l}\text { Clinical Trial } \\
\text { No. }\end{array}$ & Funding Source & $\begin{array}{l}\text { Study } \\
\text { Design }\end{array}$ & Intervention & Study Population & Study Objective \\
\hline 1. & $\begin{array}{l}\text { NCT02523183 } \\
\text { [33] }\end{array}$ & $\begin{array}{l}\text { University of Colorado, } \\
\text { Denver }\end{array}$ & $\begin{array}{l}\text { Observation } \\
\text { al Study }\end{array}$ & $\begin{array}{l}\text { Medicinal } \\
\text { Cannabis }\end{array}$ & Pediatric epilepsy patients & $\begin{array}{l}\text { To evaluate the efficacy of medical } \\
\text { cannabis on medically refractory } \\
\text { epilepsy in children. }\end{array}$ \\
\hline 2. & $\begin{array}{l}\text { NCT02397863 } \\
{[34]}\end{array}$ & $\begin{array}{l}\text { Georgia Regents } \\
\text { University, U. S. A. }\end{array}$ & $\begin{array}{l}\text { Open-Label, } \\
\text { Multicenter }\end{array}$ & $\begin{array}{l}\text { Medicinal } \\
\text { Cannabidiol }\end{array}$ & Pediatric epilepsy patients & $\begin{array}{l}\text { To evaluate the safety of Cannabidiol in } \\
\text { children suffering from epilepsy. }\end{array}$ \\
\hline 3. & $\begin{array}{l}\text { NCT02447198 } \\
{[35]}\end{array}$ & $\begin{array}{l}\text { University of Colorado, } \\
\text { Denver }\end{array}$ & $\begin{array}{l}\text { Observation } \\
\text { al Study }\end{array}$ & $\begin{array}{l}\text { Medicinal } \\
\text { Cannabidiol }\end{array}$ & Pediatric epilepsy patients & $\begin{array}{l}\text { To determine the efficacy of } \\
\text { Cannabidiol pharmacokinetics in the } \\
\text { pediatric patients with epilepsy. }\end{array}$ \\
\hline 4. & $\begin{array}{l}\text { NCT02229032 } \\
{[36]}\end{array}$ & $\begin{array}{l}\text { University of Colorado, } \\
\text { Denver }\end{array}$ & $\begin{array}{l}\text { Observation } \\
\text { al Study }\end{array}$ & $\begin{array}{l}\text { Medicinal } \\
\text { Cannabidiol } \\
(\mathrm{CBD}) \text { oil }\end{array}$ & $\begin{array}{l}\text { Uncontrolled epileptic } \\
\text { patients }\end{array}$ & $\begin{array}{l}\text { To study the antiepileptic effect of } \\
\text { cannabidioloil in a specific genetic } \\
\text { epilepsy patients. }\end{array}$ \\
\hline
\end{tabular}




\section{Conclusion}

Further investigations are required to fill the gaps between knowledge and understanding the mechanisms of action with the use of medicinal cannabis in people with epilepsy. There is a scarcity and dearth of data and knowledge regarding the practice guidelines including patient education for medicinal cannabis use in epilepsy. Without accurate, systematic data it was very challenging to determine the safety and efficacy of medicinal cannabis in epileptic patients [32]. On the other hand, evidence from laboratory studies, anecdotal reports, and small clinical studies from a number of years ago suggested that medicinal cannabis, potentially helped in controlling seizures. However, conducting studies could be difficult as researchers have limited access to cannabis due to federal regulations, lack of scientific data on the usefulness and safety of cannabis, increased financial and time constraints [9]. The use of medicinal cannabis is an ethical issue as it possessed both good and bad results- 'hindrance for getting legalized'. But, on the contrary the patient suffering has the right to access the better conventional and alternative treatment for the better qualiy of life. This factor could motivate people to black- market purchasing of medicinal cannabis. Therefore, introduction of Legislation based on internationally accepted human rights standards could prevent discrimination and rights violations, improved access to health-care services, and raise the quality of life for people with epilepsy [6]. The Food and Drug Administration is conducting a review of scientific evidence to determine whether cannabis had similar or improved effect as compared to existing treatment medications for treating epilepsy [30]. In India, the Ministry of Health and Family Welfare had proposed a National Epilepsy Program in order to, increase the public awareness about Epilepsy and fill the gap between the treatment and Quality of care in the country [7]. Thus, it could be concluded that when medicinal cannabis was used as directed in preclinical and clinical trials, the side effects were comparatively low [31].

In a nutshell, the present study focussed on medicinal cannabis and its derivatives to be further investigated for its effectiveness in treating epilepsy so that every person would get compassionate access to the treatment giving rise to a complete revolution to treat EPILEPSY.

\section{Future directions}

There is a need for more data regarding the safety and efficacy which measured the potential benefits and risks of Medicinal Cannabis and its derivatives to be used for epileptic patients by using EEG as a biomarker. Studies need to be conducted for calculating and identifying the optimal dosing, tolerability and standardization of the medicinal cannabis preparation to be used in different population suffering from epilepsy. Further, there is an urgent need for strict guidelines and regulations for standardization of medicinal cannabis in clinical research industry. Moreover, projects at regional, national as well as, international level should be implemented to fill the gap between people with epilepsy and society by proper training and education into health systems to dispel the stigma worldwide [6].

\section{Limitations of the study}

- Quality of the data collected and retrieved through searching was highly variable and conflicting.

- Included studies showed uncertainty about the doses and quality of the product composition.

- Several data were found to be missing in the included studies, like, patients' followup, number and frequency of seizure counts, length and severity of seizures.

- The cost-effectiveness of the Medicinal Cannabis intervention and the travel cost tools were not included in the study. 
South American Journal of Clinical Research

Volume 3, Issue 1, 2016

\section{References}

[1.] Anbesaw, W. , Selassie, Dulaney, A. , Wilson, Angela, M. , Malek, Janelle, L., Wagner, Smith, G. , Martz, G. , Edwards, J., Wannamaker, B. , Zack, M. M. , Koba, R. (2014). Premature Deaths Among Children with Epilepsy — South Carolina, 2000-2011. Centers for disease Control and Prevention, 63(44). Available from:

http://www. cdc. gov/mmwr/pdf/wk/mm6344. pdf.

[2.] CANNABIS RESEARCH A - Z Open Source Health Cannabis Education [Internet]. Accessed on: 2016 May 26; Available at: http://www. calgarycmmc.com/epilepsy. htm .

[3.] CDC Features: Epilepsy in Children, Citizens United for Research in Epilepsy (CURE) [Internet], 2016 April 27. Accessed on: 2016 May 27. Available from: http://www. cureepilepsy. org/news/story. asp?id=129.

[4.] ClinicalTrials. gov [Internet]. University of Colorado, Denver (United States). 2016 February 22-Identifier NCT02523183, The Use of Medicinal Cannabinoids as Adjunctive Treatment for Medically Refractory Epilepsy; Accessed on: 2016 May 25; Available from: https://clinicaltrials.

gov/ct2/show/study/NCT02523183?term=Cannabis+and+epilepsy\&rank=1.

[5.] ClinicalTrials. gov [Internet]. Georgia Regents University (United States). 2015 April 23Identifier NCT02397863, Epidiolex and Drug Resistant Epilepsy in Children (CBD); Accessed on: 2016 May 25; Available from:

https://clinicaltrials. gov/ct2/show/NCT02397863?term=Cannabis+and+epilepsy\&rank=2.

[6.] ClinicalTrials. gov [Internet]. University of Colorado, Denver (United States). 2016 February 29-Identifier NCT02447198, Cannabidiol (CBD) and Pediatric Epilepsy; Accessed on: 2016 May 25; Available from:

https://clinicaltrials. gov/ct2/show/NCT02447198?term=Cannabis + and + epilepsy\&rank=3.

[7.] ClinicalTrials. gov [Internet]. University of Colorado, Denver (United States). 2016 March 18-Identifier NCT02229032, Genetic Analysis Between Charlotte's Web Responders Versus Non- Responders in a Dravet Population; Accessed on: 2016 May 25; Available from: https://clinicaltrials. gov/ct2/show/record/NCT02229032?term=Cannabis+and+epilepsy\&rank=4.

[8.] Consroe, P. , Benedito, M. A. , Leite, J. R. , Carlini, E. A., Mechoulam, R. (1982). Effects of cannabidiol on behavioral seizures caused by convulsant drugs or current in mice. Eur J Pharmacol, 24;83(3-4):293-8. Doi: doi: http://10. 1016/0014-2999(82)90264-3. [PubMed PMID: 6129147].

[9.] Consroe, P. F., Wood, G. C. , Buchsbaum, H. (1975). Anticonvulsant nature of marihuana smoking. Journal of the American Medical Association, 234(3):306-307. Available at: http://www. cannabis-med. org/studies/ww_en_db_study_show. php?s_id=39.

[10.] Consroe, P., Wolkin, A. (1977). Cannabidiol--antiepileptic drug comparisons and interactions in experimentally induced seizures in rats. J PharmacolExpTher, 201(1):26-32. Available at:

http://www. ncbi. nlm. nih. gov/pubmed/850145?dopt=Abstract. [PubMed PMID: 850145]. [11.] Cunha, J. M. , Carlini, E. A. , Pereira, A. E. , Ramos, O. L. , Pimentel, C. , Gagliardi, R. , Sanvito, W. L. , Lander, N. , Mechoulam, R. (1980). Chronic administration of cannabidiol to healthy volunteers and epileptic patients. Pharmacology, 21(3):175-185. Available at:

http://www. cannabis-med. org/studies/ww_en_db_study_show. php?s_id=42.

[12.] Devinsky, O. , Marsh, E. , Friedman, D. , Thiele, E. , Laux, L. , Sullivan, J. , Miller, I. , Flamini, R. , Wilfong, A. , Filloux, F. , Wong, M. , Tilton, N. , Bruno, P. , Bluvstein, J. , Hedlund, J. , Kamens, R. , Maclean, J. , Nangia, S. , Singhal, N. S. , Wilson, C. A. , Patel, A., Cilio, M. R. (2005). The Lancet Neurology, 15(3), 270 - 278. DOI: http://dx. doi. org/10. 1016/S1474-4422(15)00379-8 .

[13.] Epilepsy, Fact sheet, Updated February 2016. [Internet], Accessed on: 2016 May 28; Available from: http://www. who. int/mediacentre/factsheets/fs999/en/. 
[14.] Families, Doctors, Advocates Urgently Seeking Answers in Epilepsy Deaths, Citizens United for Research in Epilepsy (CURE), [Internet], 2016 April 27. Accessed on: 2016 May 27. Available from: http://www. cureepilepsy. org/news/story. asp?id=151.

[15.] Few Answers in Understanding Death from Epilepsy, Citizens United for Research in Epilepsy (CURE), [Internet], 2016 April 27. Accessed on: 2016 May 27. Available from: http://www. cureepilepsy. org/news/story. asp?id=150.

[16.] Florek-Luszczki, M. , Wlaz, A. , Zagaja, M. , Andres-Mach, M. , Kondrat-Wrobel, M. W. , Luszczki, J. J. (2015). Effects of WIN 55, 212-2 (a synthetic cannabinoid CB1 and CB2 receptor agonist) on the anticonvulsant activity of various novel antiepileptic drugs against 6 Hz-induced psychomotor seizures in mice. PharmacolBiochem Behav. , 130:53-8. doi: http://10. 1016/j. pbb. 2015. 01. 003. [PubMed PMID: 25592741].

[17.] Gedde, M. , Maa, E. (2013). Whole Cannabis Extract of High Concentration Cannabidiol May Calm Seizures in Highly Refractory Pediatric Epilepsies. In Press with the American Epilepsy Society, 67th Annual Meeting, Available at:

https://www. thcint.com/uploads/1/9/3/7/19371199/2013_cannabidiol_may_calm_seizures. pdf.

[18.] Gedde, M. M. (2014). Clinical Experience with Cannabis in Treatment-Resistant Pediatric Epilepsy. Presentation, [Internet], Available from:

http://www. theroc. us/images/gedde_presentation. pdf.

[19.] Hadorn, D. (2003). Use of Cannabis Medicines in Clinical Practice. Internet Archive: Wayback Machine, Available from:

http://medicalmarijuana. procon. org/sourcefiles/use-of-cannabis-medicines-in-clinicalpractice. pdf.

[20.] Hellerman, C. "Federal marijuana bill would legalize some cannabis strains". CNN. Cable News Network, 2015 April 15, Accessed on: 2016 May 25; Available from:

http://edition. cnn.com/2014/07/28/health/federal-marijuana-bill/index. html.

[21.] Hamerle, M. , Ghaeni, L. , Kowski, A. , Weissinger, F. , Holtkamp, M. (2014). Cannabis and other illicit drug use in epilepsy patients. Eur J Neurol, 21(1):167-70. Available at:

http://www. cannabis-med. org/studies/ww_en_db_study_show.php?s_id=558.

[22.] Hegde, M. , Santos-Sanchez, C. , Hess, C. P. , Kabir, A. A. , Garcia, P. A. (2012). Seizure exacerbation in two patients with focal epilepsy following marijuana cessation. Epilepsy Behav, 25(4):563-6. Available at:

http://www. cannabis-med. org/studies/ww_en_db_study_show.php?s_id=560 .

[23.] Izquierdo, I. , Orsingher, O. A. , Berardi, A. C. (1973). Effect of cannabidiol and of other Cannabis sativa compounds on hippocampal seizure discharges. Psychopharmacologia, 28(1):95-102. Doi: http://link. springer.com/article/10. 1007\%2FBF00413961.

[24.] Johnson, D. D. , McNeill, J. R. , Crawford, R. D. , Wilcox, W. C. (1975). Epileptiform seizures in domestic fowl. V. The anticonvulsant activity of delta9-tetrahydrocannabinol. Can J Physiol Pharmacol., 53(6):1007-13. Available at:

http://www. ncbi. nlm. nih. gov/pubmed/1222370. [PubMed PMID: 1222370].

[25.] Jones, N. A. , Glyn, S. E. , Akiyama, S. , Hill, T. , Hill, A. J. , Weston, S. E. , Burnett, M. D. A., Yamasaki, Y. Stephens, G. J. , Whalley, B. J., Williams, C. M. (2012). Cannabidiol exerts anti-convulsant effects in animal models of temporal lobe and partial seizures. European Journal of Epilepsy, 21(5), 344 - 352.

DOI: http://dx. doi. org/10. 1016/j. seizure. 2012. 03. 001 .

[26.] Jones, N. A. , Hill, A. J. , Smith, I. , Bevan, S. A. , Williams, C. M. , Whalley, B. J. , Stephens, G. J. (2010). Cannabidiol displays antiepileptiform and antiseizure properties in vitro and in vivo. $J$ Pharmacol Exp Ther, 332(2):569-77.

doi: http://10. 1124/jpet. 109. 159145. [PubMed PMID: 19906779]; [PubMed Central PMCID: PMC2819831].

[27.] Liu, H. , Song, Z. , Liao, D. , Zhang, T. , Liu, F. , Zhuang, K. , Luo, K. , Yang, L. (2015). Neuroprotective effects of trans-caryophyllene against kainic acid induced seizure 
South American Journal of Clinical Research

Volume 3, Issue 1, 2016

activity and oxidative stress in mice. Neurochem Res, 40(1):118-23. doi: http://10. 1007/s11064-014-1474-0. [PubMed PMID: 25417010].

[28.] Megiddo, I., Colson, A. , Chisholm, D. , Dua, T. , Nandi, A. , and Laxminarayan, R. (2016). Health and economic benefits of public financing of epilepsy treatment in India: An agent-based simulation model. Epilepsia Official Journal of the International League Against Epilepsy, 57(3): 464-474. doi: http://10. 1111/epi. 13294.

[29.] Mortati, K. , Dworetzky, B. , Devinsky, O. (2007). Marijuana: an effective antiepileptic treatment in partial epilepsy? A case report and review of the literature. Rev Neurol Dis, 4(2):103-6. Available at:

http://www. cannabis-med. org/studies/ww_en_db_study_show.php?s_id=157.

[30.] Pelliccia, A. , Grassi, G. , Romano, A. , Crocchialo, P. (2005). Treatment with CBD in oily solution of drug-resistant paediatric epilepsies. Congress on Cannabis and the Cannabinoids, Leiden, The Netherlands: International Association for Cannabis as Medicine, p. 14. http://www. cannabis-med. org/studies/ww_en_db_study_show. php?s_id=173.

[31.] Philipsen, N., Butler, R. D. , Simon-Waterman, C. , Artis, J. (2014). Medical Marijuana: A Primer on Ethics, Evidence, and Politics. Journal for Nurse Practitioners, 10(9):633-640. Available at: http://www. medscape.com/viewarticle/833782_8.

[32.] Press, C. A. , Knupp, K. G. , Chapman, K. E. (2015). Parental reporting of response to oral cannabis extracts for treatment of refractory epilepsy. (2015). Epilepsy Behav, 45:49-52. Available at:http://doi:10. 1016/j. yebeh. 2015. 02. 043. [PubMed PMID: 25845492].

[33.] Rush, I. R. (2015). Initiative seeks to uncover causes, develop treatments, design possible cures for epilepsies, [Internet], Accessed on: 2016 May 27. Available from: http://articles. philly.com/2015-05-31/news/62882680_1_patients-citizens-united-seizures.

[34.] Saundra, Y. "Marijuana stops child's severe seizures. " CNN. Cable News Network. [Internet], 2013 August 7. Accessed on: 2016 May 25. Available from:

http://edition. cnn.com/2013/08/07/health/charlotte-child-medical-marijuana.

[35.] Schachter, S. C. , Shafer, P. O. , Sirven, J. I. (2013). Treating Seizures and Epilepsy. Epilepsy Foundation, [Internet], Available at:

http://www.epilepsy.com/learn/treating-seizures-and-epilepsy/other-treatment-

approaches/medical-marijuana-and-epilepsy\%2024.

[36.] Shirazi-zand, Z., Ahmad-Molaei, L., Motamedi, F., Naderi, N. (2013). The role of potassium BK channels in anticonvulsant effect of cannabidiol in pentylenetetrazole and maximal electroshock models of seizure in mice. Epilepsy Behav. 28(1):1-7. doi: http://10. 1016/j. yebeh. [PubMed PMID: 23644464]. 